\title{
Exploring difference and overlap between schizophrenia, schizoaffective and bipolar disorders using resting-state brain functional networks
}

\author{
Yuhui Du*, Jingyu Liu, Member IEEE, Jing Sui, Member IEEE, Hao He, Godfrey D Pearlson \\ and Vince D. Calhoun, Fellow IEEE
}

\begin{abstract}
Schizophrenia, schizoaffective and bipolar disorders share some common symptoms. However, the biomarkers underlying those disorders remain unclear. In fact, there is still controversy about the schizoaffective disorder with respect to its validity of independent category and its relationship with schizophrenia and bipolar disorders. In this paper, based on brain functional networks extracted from resting-state fMRI using a recently proposed group information guided ICA (GIG-ICA) method, we explore the biomarkers for discriminating healthy controls, schizophrenia patients, bipolar patients, and patients with two symptom defined subsets of schizoaffective disorder, and then investigate the relationship between different groups. The results demonstrate that the discriminating regions mainly including frontal, parietal, precuneus, cingulate, supplementary motor, cerebellar, insular and supramarginal cortices perform well in distinguishing the different diagnostic groups. The results also suggest that schizoaffective disorder may be an independent disorder, although its subtype characterized by depressive episodes shares more similarity with schizophrenia.
\end{abstract}

\section{INTRODUCTION}

Schizophrenia (SZ), bipolar disorder (BP), and schizoaffective disorder (SAD) have overlapping symptoms, shared risk genes and co-occurrence within relatives ${ }^{[1,2]}$. SZ is characterized by altered perception, loss of motivation, and impairment in multiple cognitive domains. BP is marked by alternating episodes of mania and depression; some patients exhibit psychotic symptoms. SAD is diagnosed when symptom criteria for both SZ and BP are met. Among SAD, one type is characterized by manic, hypomanic or mixed episodes; the other is determined when the individual has depressive episodes exclusively.

Recently, there has been increasing interest in identifying biomarkers of SZ, BP and SAD based on functional networks extracted from neuroimaging data, which is expected to provide adjunctive information for clinic diagnosis. Using oddball-task fMRI data, Garrity ${ }^{[3]}$ found significant difference between SZ and healthy controls (HC) in the default mode network (DMN). Whitfield ${ }^{[4]}$ found that SZ patients and their

* Research supported by NIH.

Yuhui Du is with The Mind Research Network, Albuquerque, NM, 87106, USA and School of Information and Communication Engineering, North University of China, Taiyuan, 030051, China. (corresponding author phone: 505-573-1356, e-mail: ydu@mrn.org and duyuhui@nuc.edu.cn )

Jingyu Liu, Jing Sui, Hao He and Vince D. Calhoun are with The Mind Research Network and Dept. of ECE of New Mexico, Albuquerque, NM, 87106, USA. (vcalhoun@unm.edu).

Godfrey D Pearlson is with Dept. of Psychiatry, Yale University, New Haven, CT, 06519, USA. (godfrey.pearlson@yale.edu)

U.S. Government work not protected by U.S. copyright first-degree relatives exhibited significantly reduced task-related suppression in medial prefrontal cortex. Ongur ${ }^{[5]}$ applied independent component analysis (ICA) to resting-state fMRI of SZ including a few SAD, BP and HC; data suggested that both SZ and BP showed reduced connectivity in the medial prefrontal cortex of the DMN. Khadka ${ }^{[6]}$ analyzed fMRI data of SZ, BP and their unaffected first-degree relatives using ICA, and found significant difference in seven functional networks. Argyelan ${ }^{[7]}$ studied whole brain networks using a region-of-interest (ROI) based method, determining that SZ had lower functional connectivity than $\mathrm{HC}$, whereas $\mathrm{BP}$ had functional connectivity intermediate to SZ and $\mathrm{HC}$. Therefore, exploring functional networks based biomarkers for diagnosis of SZ, BP, and SAD can be potentially fruitful.

There is also controversy about SAD's validity as an independent category as well as its relationship to SZ and BP. Different opinions exist: (1) SAD is a independent diagnosis ${ }^{[1,2]}$. (2) SAD is an disease intermediate between SZ and $\mathrm{BP}^{[8,9]}$. (3) $\mathrm{SAD}$ represents the co-morbidity of schizophrenia and bipolar disorder ${ }^{[10]}$. (4) SAD is an atypical form of schizophrenia or bipolar disorder ${ }^{[11-13]}$. (5) SAD is a heterogeneous group composed by both $\mathrm{SZ}$ and $\mathrm{BP}^{[14]}$. In sum, no consensus about SAD has been reached. Therefore, investigating the relationship between SZ, BP and SAD based on brain functional networks extracted from resting-state fMRI is necessary.

Although ICA has been widely used in brain functional network extraction, it is difficult to obtain individual networks with correspondence across subjects due to the random order of its resulting independent components (ICs). In this study, we use a variant of group $\mathrm{ICA}^{[15]}$ called group information guided independent component analysis (GIG-ICA) ${ }^{[16]}$ to accurately capture the individual functional networks and simultaneously preserve correspondence of networks across subjects. The method uses a new one-unit ICA with spatial reference approach to reconstruct the subject-specific maps enabling a single-subject optimization of independence while still using the group model to preserve the correspondence of the components across subjects. GIG-ICA enables us to capture the probable similarity and the subtle differences in functional networks expected for SZ, BP, and SAD.

\section{MATERIALS AND METHODS}

\section{A. Materials}

Resting-state fMRI data of $20 \mathrm{HC}, 20 \mathrm{SZ}, 20 \mathrm{BP}, 20 \mathrm{SAD}$ with manic episode (SADM) and 13 SAD with depressive episode exclusively (SADD) scanned at the Olin Neuropsychiatry Research Center were analyzed (see Table 1). 
Scans were acquired on a 3T dedicated head scanner (Siemens Allegra) using gradient echo planar imaging (EPI) with parameters: repeat time $(\mathrm{TR})=1.5 \mathrm{~s}$, echo time $(\mathrm{TE})=27 \mathrm{~ms}$, field of view $=24 \mathrm{~cm}$, acquisition matrix $=64 \times 64$, flip angle $=60^{\circ}$, voxelsize $=3.44 \mathrm{~mm} \times 3.44 \mathrm{~mm} \times 5 \quad \mathrm{~mm}, \quad$ slice thickness $=5 \mathrm{~mm}$, number of slices $=29$.

TABLE 1. DEMOGRAPHIC CHARACTERISTICS

\begin{tabular}{|c|c|c|c|c|c|}
\hline Subjects & HC & SZ & BP & SADM & SADD \\
\hline Mean age (years) & 33.9 & 28.8 & 31.2 & 35.2 & 39.9 \\
\hline Male percent & $50 \%$ & $65 \%$ & $40 \%$ & $40 \%$ & $20 \%$ \\
\hline
\end{tabular}

\section{B. Methods}

fMRI data were preprocessed using SPM8. The first ten volumes were discarded, and the remaining images were slice-time corrected and realigned to the first volume for head-motion correction. Subsequently, the images were spatially normalized to the MNI EPI template, resliced to $3 \mathrm{~mm} \times 3 \mathrm{~mm} \times 3 \mathrm{~mm}$ voxels, and smoothed with a Gaussian kernel with a full-width at half-maximum (FWHM) of $8 \mathrm{~mm}$.

The preprocessed fMRI data of all subjects were subjected to GIG-ICA for extracting their subject-specific functional networks. (1) Group level ICA ${ }^{[17,18]}$ was applied to all subjects' datasets to compute group ICs, which includes two levels (subject level and group level) principal component analysis (PCA) applied to the temporally concatenated data and ICA applied to the dimensionality reduced data using Infomax algorithm. The number of ICs was set to 30, and ICASSO technique ${ }^{[19]}$ with 20 ICA runs was used to get reliable group ICs. (2) Artifact group ICs were identified manually based on the group ICs and the related individual time courses (TCs). (3) The non-artifact group ICs were taken as references in a one-unit ICA with spatial reference using a multi-objective optimization solver to compute the subject-specific networks ${ }^{[16]}$.

To detect regions for distinguishing those disorders, we analyzed the obtained subject-specific function networks as follows. First, statistical map was created with voxel-wise one-sample t-tests ( $p<0.01$ with FDR correction) on IC values of all subjects for each network. Then, network spatial maps in each statistically significant voxel of all subjects were entered into a voxel-wise five-level one-way ANCOVA with age and gender as covariates $(p<0.05)$ for each network. Subsequently, based on the voxels showing a main effect of group difference extracted from ANCOVA, support vector machine recursive feature elimination (SVM-RFE) was applied to extract discriminating regions. Finally, for each discriminating region, mean Z-score among voxels within the region was calculated for each subject, then the mean Z-scores of different subjects were compared between any pair of groups using two-sample t-tests ( $p<0.01$ with FDR correction).

Based on those discriminating regions, projection and clustering methods were performed to investigate the relationship between different groups and examine the discrimination ability. For each subject, IC values within all those discriminating regions were concatenated as a feature vector. Based on the features, the similarity $S$ between any pair of subjects was obtained by computing Pearson correlation coefficient between their features, accordingly, the distance between any pair of subjects was specified as $1-S$. Therefore, distance matrix or similarity matrix (size: $93 * 93$ ) reflecting the distance or similarity between all subjects can be obtained. To investigate the overall relationship between groups, we averaged the values in each sub-block of the distance matrix. To visualize the relationship between subjects, a projection method named t-Distributed Stochastic Neighbor Embedding (t-SNE) ${ }^{[20]}$ was applied to project feature vectors from all subjects onto a 2D plane. Then, clustering approaches including K-Means with 100 times implementation, normalized cuts (Ncut) ${ }^{[21]}$, and hierarchical clustering were performed separately to cluster those feature vectors. The cluster number was specified as 5 to evaluate the ability to capture the 5 pre-defined groups.

\section{RESULTS}

\section{A. Brain functional networks extracted using GIG-ICA}

Based on the preprocessed resting-state fMRI data of 93 subjects, 30 group ICs were obtained. Then, 18 artifact group ICs were detected and removed via manual inspection. Subsequently, 12 individual networks were estimated for each subject. One-sample t-tests results (FDR corrected, $\mathrm{p}<0.01$ ) of networks are shown in Fig. 1, and these networks included fronto-parietal networks (IC 1 and IC 2), default mode networks (IC 3, IC 5, and IC 6), salience network (IC 4), auditory related network (IC 8), parietal network (IC 7), vision related networks (IC 9), visuospatial network (IC 10), cerebellum (IC 11), and sensory-motor network (IC 12).

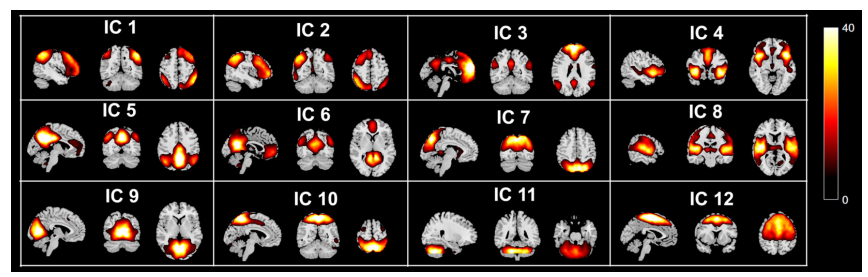

Figure 1. One-sample t-tests statistics (FDR corrected, $\mathrm{p}<0.01$ ) of 12 functional networks.

\section{B. Discriminating regions extracted from functional networks}

Fig. 2 shows primary discriminating regions with cluster size $>50$ extracted from functional networks. As summarized in Table 2, those regions mainly involved frontal, parietal, precuneus, cingulate, supplementary motor, cerebellar, insular, and supramarginal cortices.

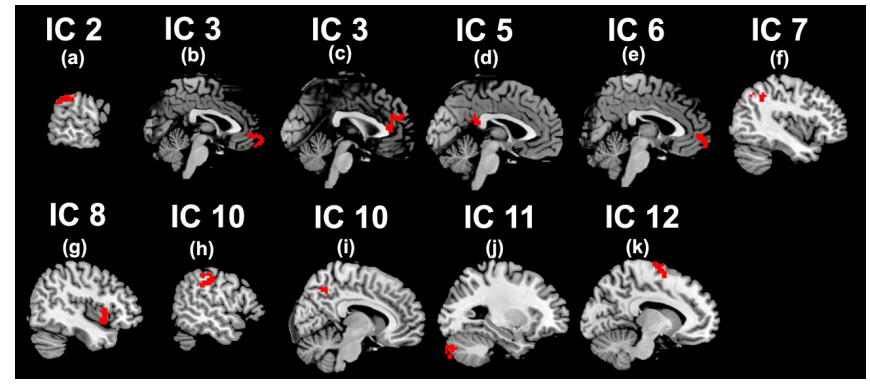

Figure 2. Primary discriminating regions extracted from 12 functional networks. Their related networks (ICs) are also denoted. The regions (a)-(k) are also reported in Table 2. 
TABLE 2. PARIMARY DISCRIMINATING REGIONS EXTRACTED FROM FUNCTIONAL NETWORKS, ITS VOLUME IN CUBIC MILLIMETERS, AND ITS CORRESPONDING MONTREAL NEUROLOGICAL INSTITUTE COORDINATES

\begin{tabular}{|c|c|c|c|c|}
\hline IC & $\begin{array}{c}\text { Region } \\
\text { ID }\end{array}$ & Region (L/R) & $\begin{array}{c}\text { Volume } \\
\mathbf{( m m}^{\mathbf{3}} \mathbf{)}\end{array}$ & $\mathbf{( x , \mathbf { y } , \mathbf { z } )}$ \\
\hline IC 2 & (a) & SupraMarginal (L) & 1431 & $(-62,-40,34)$ \\
\hline IC 3 & (b) & Frontal_Med_Orb (R) & 2052 & $(2,64,-8)$ \\
\hline IC 3 & (c) & Cingulate_Ant (L) & 2484 & $(-1,42,23)$ \\
\hline IC 5 & (d) & Cingulate_Post (L) & 1674 & $(-2,-43,22)$ \\
\hline IC 6 & (e) & Frontal_Med_Ord (R) & 1431 & $(3,58,-4)$ \\
\hline IC 7 & (f) & Parietal_Inf (L) & 1404 & $(-41,-49,41)$ \\
\hline IC 8 & (g) & Insula (R) & 1674 & $(44,11,4)$ \\
\hline IC 10 & (h) & Parietal_Inf (L) & 1404 & $(-54,-25,40)$ \\
\hline IC 10 & (i) & Precuneus (R) & 3078 & $(8,-57,48)$ \\
\hline IC 11 & (j) & Cerebellum_Crus2 (L) & 1674 & $(-28,-80,-35)$ \\
\hline IC 12 & (k) & Supp_Motor_Area (L) & 4374 & $(-11,-5,74)$ \\
\hline
\end{tabular}

Based on each discriminating region, the mean Z-score in the related functional network among voxels within the region was calculated for each subject (Fig. 3). For each region, the mean Z-scores of different subjects were then compared between any pair of groups using two-sample t-tests. It is observed that different regions represented different inter-group relationship, reflecting the complexity of those disorders. In the next section, we used features from all those discriminating regions to explore the relationship between groups.
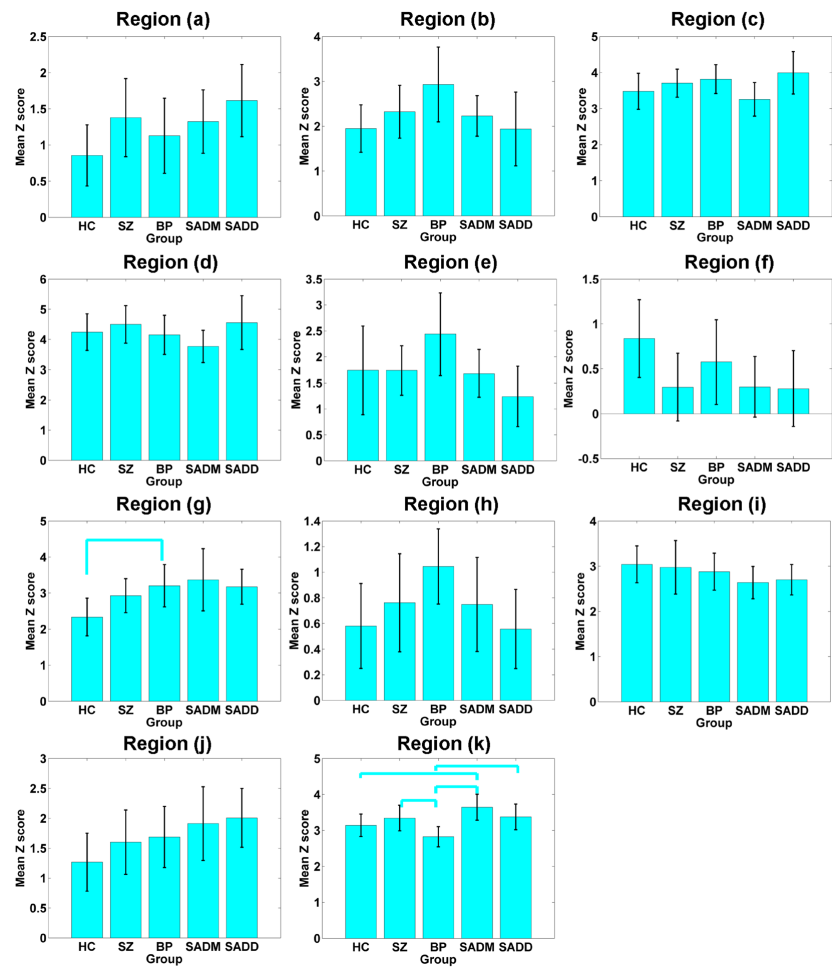

Figure 3. The bar plot of mean Z-score across subjects for each region for $\mathrm{HC}$, SZ, BP, SADM, and SADD, separately. The two-sample t-tests between any pair of groups that survived $\mathrm{p}<0.01$ with FDR correction are denoted using lines. The regions (a)-(k) are corresponding to regions reported in Figure 2 and Table 2 .

\section{Relationship between groups}

To investigate the relationship between groups, IC values within all discriminating regions were concatenated as a feature vector for each subject. As shown in Fig. 4(A), the distance matrix between feature vectors from all subjects illustrates that subjects in the same group showed more similarity than others. Fig. 4(B) displays the mean distance matrix. SADM group and SADD group were the most similar to each other. For SZ group, SADD group was the closest group. Projection result using t-SNE (Fig. 5) shows that different groups were well separated, although some groups had great affinity or overlap. The center of the projected points from SADM group and that of SADD group were closer with each other than with other groups, which is consistent with that both SADM and SADD belong to SAD. From Fig. 5, we also find that BP group was closer to $\mathrm{HC}$ group than other groups, which indicates BP patients had more similar functional network pattern to $\mathrm{HC}$ than the patients with the other disorders. The clustering results of all subjects using K-Means, Ncut and hierarchical clustering are shown in Fig. $6(A)-(C)$, which demonstrate that subjects from the same group can be well clustered, indicating that the discriminating regions are reasonable. The linkage result from hierarchical clustering is shown in Fig. 6(D), which reflects how subjects were clustered into groups, thus reflects the relationship between groups. When the cluster number was set to 4, SADM and SADD were clustered into one cluster. When the cluster number was set to 3, SADM, SADD and SZ belonged to one cluster. When the cluster number was set to 2 , one cluster included SADM, SADD and SZ, and the other cluster included $\mathrm{HC}$ and BP. Therefore, the conclusion from Fig. 6(D) is consistent to the above.
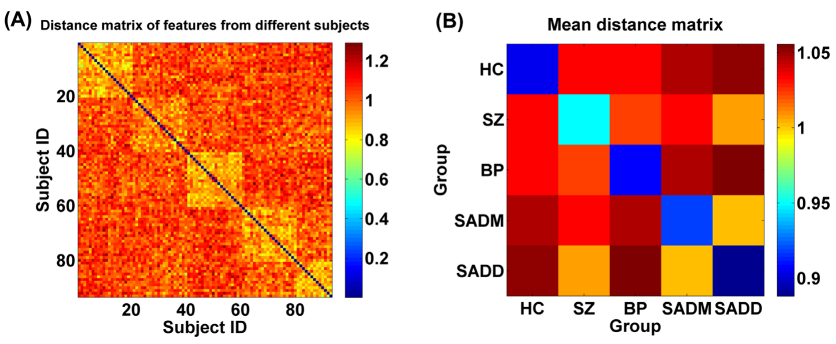

Figure 4. (A) The distance matrix between the feature vectors from all 93 subjects, and the $\mathrm{x}$ and $\mathrm{y}$ axis denote the subject ID. Subject ID 1-20 denote HC, subject ID 21-40 denote SZ, subject ID 41-60 denote BP, subject ID 61-80 denote SADM, and subject ID 81-93 denote SADD. (B) The mean distance matrix obtained by averaging the values in each sub-block of the distance matrix.

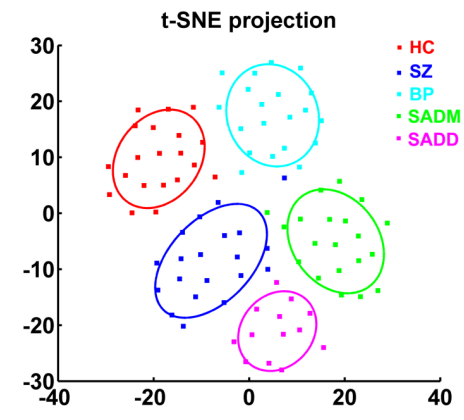

Figure 5. The projection results using t-SNE of all 93 subjects. Different points denote different subjects, and different colors denote different groups. Each ellipse reflects center (mean) and standard deviation for one group. 


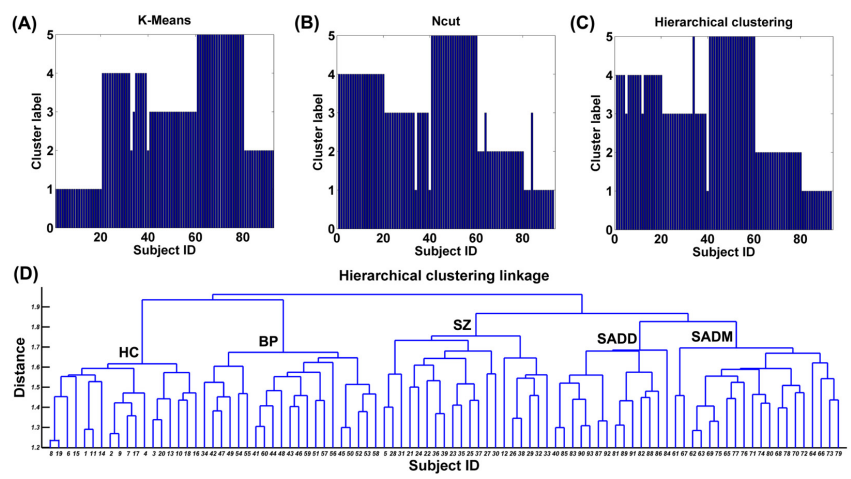

Figure 6. Clustering results of all 93 subjects obtained from (A) K-Means, (B) Ncut and (C) hierarchical clustering. (D) The linkage result from hierarchical clustering. Subject ID 1-20 denote HC, subject ID 21-40 denote SZ, subject ID 41-60 denote BP, subject ID 61-80 denote SADM, and subject ID 81-93 denote SADD.

\section{DISCUSSIONS AND CONCLUSIONS}

$\mathrm{SZ}, \mathrm{BP}$, and SAD share some common symptoms, however, the biomarkers underlying their distinct neural mechanisms remain unclear. In fact, there is still a controversy about SAD's independence as well as the relationship among these disorders. Exploring specific vs shared biomarkers of each disorder and the relationship among disorders from a functional network perspective is promising. In this study, we extracted brain functional networks using a recently proposed GIG-ICA method from resting-state fMRI data of $\mathrm{HC}, \mathrm{SZ}, \mathrm{BP}$, and two types of SAD, and then explored the discriminating regions from functional networks, subsequently investigated the relationship between different groups and examined the discrimination ability of the founded biomarkers. The results demonstrate that in general, subjects from the same group have similar functional network patterns. SADM and SADD are the most similar groups to each other, which is consistent with these two subtypes both belonging to SAD. BP has more similarity to $\mathrm{HC}$ than other groups, which accords with that bipolar disorder has less impaired performance than the other two disorders. SADD group shares high similarity with SZ group in functional network pattern. Data from SZ, SADM and SADD groups resemble one another while $\mathrm{BP}$ and $\mathrm{HC}$ groups are close, in agreement with SAD being classified in the same diagnostic class as SZ in the Diagnostic and Statistical Manual of Mental Disorders (DSM-IV-TR). The results suggest that $\mathrm{SAD}$ is an independent disorder although SADD has high similarity with SZ. In addition, the study finds that the discriminating regions mainly comprise frontal, parietal, precuneus, cingulate, supplementary motor, cerebellar, insular, and supramarginal cortices, which can result in good performance for discriminating the four disorder and healthy control groups.

\section{ACKNOWLEDGMENT}

This work was partially supported by National Institutes of Health grants R01EB006841, National Sciences Foundation grants 1016619, the Centers of Biomedical Research Excellence (COBRE) grant 5P20RR021938/P20GM103472 (VDC), and National Institute of Mental Health (NIMH) Grant R37MH43775 (GDP).

\section{REFERENCES}

[1] D. Malaspina, et al., "Schizoaffective Disorder in the DSM-5," Schizophr Res, vol. 150(1), pp. 21-25, 2013.

[2] V. E. Cosgrove and T. Suppes, "Informing DSM-5: biological boundaries between bipolar I disorder, schizoaffective disorder, and schizophrenia," BMC Med, 11: 127, 2013.

[3] A. G. Garrity, G. D. Pearlson, K. McKiernan, D. Lloyd, K. A. Kiehl and V. D. Calhoun, "Aberrant "default mode" functional connectivity in schizophrenia," Am J Psychiatry, vol. 164(3), pp. 450-457, 2007.

[4] S. Whitfield-Gabrieli, et al., "Hyperactivity and hyperconnectivity of the default network in schizophrenia and in first-degree relatives of persons with schizophrenia," Proc Natl Acad Sci U S A, vol. 106(4), pp. 1279-1284, 2009.

[5] D. Ongur, et al., "Default mode network abnormalities in bipolar disorder and schizophrenia," Psychiatry Res, vol. 183(1), pp. 59-68, 2010.

[6] S. Khadka, S. A. Meda, M. C. Stevens, D. C. Glahn, V. D. Calhoun, J. A. Sweeney, C. A. Tamminga, M. S. Keshavan, K. O'Neil, D. Schretlen and G. D. Pearlson, "Is aberrant functional connectivity a psychosis endophenotype? A resting state functional magnetic resonance imaging study," Biol Psychiatry, vol. 74(6), pp. 458-466, 2013.

[7] M. Argyelan, T. Ikuta, P. DeRosse, R. J. Braga, K. E. Burdick, M. John, P. B. Kingsley, A. K. Malhotra and P. R. Szeszko, "Resting-state FMRI connectivity impairment in schizophrenia and bipolar disorder," Schizophr Bull, vol. 40(1), pp. 100-110, 2014.

[8] S. Gupta, C. H. Steinmeyer, K. Lockwood, B. Lentz and K. Schultz, "Comparison of older patients with bipolar disorder and schizophrenia/schizoaffective disorder," Am J Geriatr Psychiatry, vol. 15(7), pp. 627-633, 2007.

[9] E. Cheniaux, et al., "Does schizoaffective disorder really exist? A systematic review of the studies that compared schizoaffective disorder with schizophrenia or mood disorders," J Affect Disord, vol. 106(3), pp. 209-217, 2008.

[10] T. M. Laursen, E. Agerbo and C. B. Pedersen, "Bipolar disorder, schizoaffective disorder, and schizophrenia overlap: a new comorbidity index," J Clin Psychiatry, vol. 70(10), pp. 1432-1438, 2009.

[11] A. M. Bogan, E. S. Brown and T. Suppes, "Efficacy of divalproex therapy for schizoaffective disorder," J Clin Psychopharmacol, vol. 20(5), pp. 520-522, 2000.

[12] E. Cascade, A. H. Kalali and P. Buckley, "Treatment of schizoaffective disorder," Psychiatry (Edgmont), vol. 6(3), pp. 15-17, 2009.

[13] C. R. Lake and N. Hurwitz, "Schizoaffective disorders are psychotic mood disorders; there are no schizoaffective disorders," Psychiatry Res, vol. 143(2-3), pp. 255-287, 2006.

[14] J. J. Levitt and M. T. Tsuang, "The heterogeneity of schizoaffective disorder: implications for treatment," Am J Psychiatry, vol. 145(8), pp. 926-936, 1988.

[15] V. D. Calhoun and T. Adali, "Multisubject independent component analysis of fMRI: a decade of intrinsic networks, default mode, and neurodiagnostic discovery," IEEE Rev Biomed Eng, vol. 5, pp. 60-73, 2012.

[16] Y. H. Du and Y. Fan, "Group information guided ICA for fMRI data analysis," Neuroimage, vol. 69, pp. 157-197, 2013.

[17] V. D. Calhoun, T. Adali, G. D. Pearlson and J. J. Pekar, "A method for making group inferences from functional MRI data using independent component analysis," Hum Brain Mapp, vol. 14(3), pp. 140-151, 2001.

[18] E. B. Erhardt, S. Rachakonda, E. J. Bedrick, E. A. Allen, T. Adali and V. D. Calhoun, "Comparison of multi-subject ICA methods for analysis of fMRI data," Hum Brain Mapp, vol. 32(12), pp. 2075-2095, 2011.

[19] J. Himberg, A. Hyvarinen and F. Esposito, "Validating the independent components of neuroimaging time series via clustering and visualization," Neuroimage, vol. 22(3), pp. 1214-1222, 2004.

[20] L. van der Maaten and G. Hinton, "Visualizing Data using t-SNE," Journal of Machine Learning Research, vol. 9, pp. 2579-2605, 2008.

[21] J. B. Shi and J. Malik, "Normalized cuts and image segmentation," IEEE Transactions on Pattern Analysis and Machine Intelligence, vol. 22(8), pp. 888-905, 2000. 